\title{
ON THE PROBLEMS OF DETERMINING KNOTS BY THEIR COMPLEMENTS AND KNOT COMPLEMENTS BY THEIR GROUPS
}

\begin{abstract}
JONATHAN SIMON 1
ABSTRACT. Modulo the conjecture that the complement of a prime knot in the 3-sphere is determined by its fundamental group, we show that at most finitely many mutually inequivalent knots can have homeomorphic complements.
\end{abstract}

In this note, we shall exhibit relationships between the following well-known conjectures about tame knots in the 3-sphere. Our main result is that, modulo the Complements conjecture, given four knots with homeomorphic complements, at least two of the knots must be equivalent.

KNOT TyPe CONJECTURE. If $K$ and $L$ are knots with homeomorphic complements, then $K$ and $L$ are equivalent knots (in the sense that there exists a homeomorphism $f: S^{3} \rightarrow S^{3}$ such that $\left.f(K)=L\right)$.

Knot Complements Conjecture. If $K$ and $L$ are prime knots with isomorphic knot groups then $S^{3}-K$ and $S^{3}-L$ are homeomorphic.

The Type conjecture has been established, in a somewhat stronger form, for many knots ([1], [3], [7]-[10], [13], [14]). The Complements conjecture became approachable with the advent of the Annulus Theorem and the Torus Theorem [5], [6], [15]. It follows from Theorem 10 of [6], Lemmas 2.1 and 2.2 of [12], and the main result of [2], that if $K$ and $L$ are counterexamples to the Complements conjecture, then each is a nontrivial cable knot; using the notation of $\left[12\right.$, p. 2], $K=J\left(p, q ; K_{1}\right)$ and $L=J\left(r, s ; L_{1}\right)$ for some nontrivial knots $K_{1}, L_{1}(|q|,|s| \geqslant 2)$.

For a knot $K$, let $C^{3}(K)$ denote the closed complement of a regular neighborhood of $K$ and let $\left(\mu_{K}, \lambda_{K}\right)$ be a meridian-longitude pair for $K$. Suppose $L$ is a knot such that $C^{3}(K)$ is homeomorphic to $C^{3}(L)$. If $f: C^{3}(K) \rightarrow C^{3}(L)$ is a homeomorphism then, for homology reasons, $f\left(\lambda_{K}\right)$ $=\lambda_{L}^{ \pm 1}$ and, for some integer $n, f\left(\mu_{K}\right)=\mu_{L}^{ \pm 1} \lambda_{L}^{n}$. If $n=0$, then $f$ extends to a homeomorphism of $S^{3}$ and we can conclude that $K$ and $L$ are equivalent. Modulo the Complements conjecture, we can reach the same conclusion if we are given any homeomorphism $f$ with $|n| \neq 1,2$, or 4 .

Theorem 1 (Modulo the Knot Complements Conjecture). If $K$ and $L$ are knots with homeomorphic complements, and there exists a homeomorphism

Presented to the Society, July 24, 1975; received by the editors August 5, 1975.

AMS (MOS) subject classifications (1970). Primary 55A25, 57A10.

${ }^{1}$ Partially supported by NSF Grant GP29430. 
$f: C^{3}(K) \rightarrow C^{3}(L)$ such that $f\left(\mu_{K}\right)=\mu_{L}^{ \pm 1} \lambda_{L}^{n}$, where $|n| \neq 1,2$, or 4 , then $K$ and $L$ are equivalent knots.

Proof. Suppose such a homeomorphism exists. If $n=0$, then, as noted above, $f$ extends to a homeomorphism of $S^{3}$ taking $K$ to $L$. We assume now that $n \neq 0$. Orient the curves $\mu_{L}, \lambda_{L}$ so that $f\left(\lambda_{K}\right)=\lambda_{L}$ and $f\left(\mu_{K}\right)=\mu_{L} \lambda_{L}^{m}$, $|m| \neq 0,1,2$, or 4 .

If $J$ is a $(p, q)$-curve on $\partial C^{3}(K)$ (i.e., $\left.J \sim p \mu_{K}+q \lambda_{K}\right), f(J)$ is a $(p, m p+q)$ curve on $\partial C^{3}(L)$. If $m$ is even, let $p=1$ and $q=-m / 2$; if $m$ is odd, let $p=2$ and $q=-m$. We then have $p$ and $q$ relatively prime, $|q| \geqslant 3$, and $m p+q$ $=-q$.

Since $f$ maps the curve $\mu_{k}^{p} \lambda_{k}^{q}$ to the curve $\mu_{L}^{p} \lambda_{L}^{-q}$, the induced isomorphism $f_{*}: \pi_{1}\left(C^{3}(K)\right) \rightarrow \pi_{1}\left(C^{3}(L)\right)$ can be extended to an isomorphism of amalgamated free products

$$
\pi_{1}\left(C^{3}(K)\right)_{\mu k \lambda_{k}^{k}=t^{q}}^{*}\langle t\rangle \cong \pi_{1}\left(C^{3}(L)\right)_{\mu \mathcal{L} \lambda_{L}^{-q}=s^{-q}}^{*}\langle s\rangle
$$

by sending $t$ to $s^{-1}$. In other words, the cable knots $J(p, q ; K)$ and $J(p,-q ; L)$ have isomorphic groups. Assuming the Complements conjecture, we then see that $J(p, q ; K)$ and $J(p,-q ; L)$ have homeomorphic complements. But since $|q| \geqslant 3,|q|$-strand cable knots have "Property P" [7], [14], so we conclude that $J(p, q ; K)$ and $J(p,-q ; L)$ are equivalent knots. It follows (see [4, p. 10]) that $J(p, q ; K)$ has the same isotopy type as $J(p,-q ; L)$ or its mirror image (which is a cable about the mirror image of $L$ ) and so (Theorem 21.5 of [11]) $K$ has the same isotopy type as $L$ or its mirror image, i.e., $K$ and $L$ are equivalent.

In all of the references cited earlier, the classes of knots for which the Type conjecture holds have been shown to have a stronger property. The following definition of "Property P" is equivalent to the original definition given in [1].

Definition. For a given integer $n \geqslant 1$, a knot $K$ has Property $\mathrm{P}_{\eta}$ if neither of the manifolds obtained by attaching 2-handles ("pillboxes") to $C^{3}(K)$ along $( \pm 1, n)$ curves on $\partial C^{3}(K)$ is simply connected. We say $K$ has Property $\mathrm{P}$ if $K$ has Property $\mathrm{P}_{n}$ for each $n$.

If it can be shown that all knots have Property $\mathrm{P}_{n}$ for $n=1,2$, and 4 , then Theorem 1 will say that the Complements conjecture implies the Type conjecture. In the proof of Theorem 1, we cannot handle the case $|n|=4$ since it is still unknown whether 2-strand cable knots are determined by their complements. By Theorem 2(c) of [14], Property $P_{1}$ is enough to conclude that cable knots have Property $\mathrm{P}$. We thus have the following variation on Theorem 1.

Theorem 2 (Modulo the Complements Conjecture). If all nontrivial knots have Property $\mathrm{P}_{n}$ for $n=1$ and 2, then the Type conjecture is a theorem.

Our final result indicates that any proof of the Complements conjecture would have to contain a proof of a weak form of the Type conjecture, namely that there is an upper bound to the number of mutually inequivalent knots all with the same complement.

Theorem 3 (Modulo the Complements Conjecture). If $K_{i}(i=1,2,3,4)$ 
are knots with homeomorphic complements, then at least two of these knots are equivalent.

Proof. For $i=1,2,3,4$, let $\left(\mu_{i}, \lambda_{i}\right)$ be a meridian-longitude pair for $K_{i}$ and for $i=1,2,3$, let $f_{i}: C^{3}\left(K_{i}\right) \rightarrow C^{3}\left(K_{i+1}\right)$ be a homeomorphism. Orient the curves $\mu_{i}, \lambda_{i}$ so that for some integers $n_{i}, f_{i}\left(\mu_{i}\right)=\mu_{i+1} \lambda_{i+1}^{n_{i}}$ and $f_{i}\left(\lambda_{i}\right)=\lambda_{i+1}(i$ $=1,2,3)$. For each $i, j(1 \leqslant i<j \leqslant 4)$, let $g_{i j}: C^{3}\left(K_{i}\right) \rightarrow C^{3}\left(K_{j}\right)$ be the homeomorphism obtained by composing the $f_{i}$. It is easy to show that there do not exist integers $a, b$, and $c$, such that $\{a, b, c, a+b, b+c, a+b+c\}$ $\subseteq\{ \pm 1, \pm 2, \pm 4\}$. Therefore, for some $i<j, g_{i j}$ satisfies the hypothesis of Theorem 1, and so we can conclude that $K_{i}$ and $K_{j}$ are equivalent.

\section{REFERENCES}

1. R. H. Bing and J. M. Martin, Cubes with knotted holes, Trans. Amer. Math. Soc. 155 (1971), 217-231. MR 43 \# 4018a.

2. G. Burde and H. Zieschang, Eine Kennzeichnung der Torusknoten, Math. Ann. 167 (1966), 169-176. MR 35 \# 1008.

3. A. Connor, Splittable knots, Ph.D. Thesis, Univ. of Georgia, Athens, Ga., 1969.

4. R. H. Crowell and R. H. Fox, Introduction to knot theory, Ginn, Boston, Mass., 1963. MR $26 \# 4348$.

5. C. Feustel, Embeddings and essential embeddings of annuli and möbius bands in $M^{3}$ (preprint).

6. - The torus theorem and its applications (preprint).

7. F. González-Acuña, Dehn's construction on knots, Bol. Soc. Mat. Mexicana (2) 15 (1970), 58-79. MR 50 \#8495.

8. J. Hempel, A simply connected 3-manifold is $S^{3}$ if it is the sum of a solid torus and the complement of a torus knot, Proc. Amer. Math. Soc. 15 (1964), 154-158. MR 28 \# 599.

9. J. Neuzil, Surgery on a curve in a solid torus, Trans. Amer. Math. Soc. 204 (1975), 385-406.

10. D. Noga, Über den Aussenraum von Produktknoten und die Bedeutung der Fixgruppen, Math. Z. 101 (1967), 131-141. MR 36 \#2137.

11. H. Schubert, Knoten und Vollringe, Acta Math. 90 (1953), 131-286. MR 17, 291.

12. J. Simon, An algebraic classification of knots in $S^{3}$, Ann. of Math. (2) 97 (1973), 1-13.

13. - On knots with nontrivial interpolating manifolds, Trans. Amer. Math. Soc. 160 (1971), 467-473. MR 44 \#5949.

14. - Some classes of knots with property P, Topology of Manifolds (Proc. Inst., Univ. of Georgia, Athens, Ga., 1969), Markham, Chicago, Ill., 1970, pp. 195-199. MR 43 \# 4018b.

15. F. Waldhausen, On the determination of some bounded 3-manifolds by their fundamental groups alone, Proc. Internat. Sympos. Topology and Appl. (Herzog-Novi, Yugoslavia, 1968), Beograd, 1969, pp. 331-332.

Department of Mathematics, University of Iowa, Iowa City, Iowa 52242 